Published in final form as:

Qin, Q., Heil, T., Antonietti, M., \& Oschatz, M. (2018). Single-Site Gold Catalysts on Hierarchical N-Doped Porous Noble Carbon for Enhanced Electrochemical Reduction of Nitrogen. Small Methods, 2(12): 1800202. doi:10.1002/smtd.201800202.

\title{
Single-Site Gold Catalysts on Hierarchical N-Doped Porous Noble Carbon for Enhanced Electrochemical Reduction of Nitrogen
}

Qing Qin, Tobias Heil, Markus Antonietti and Martin Oschatz

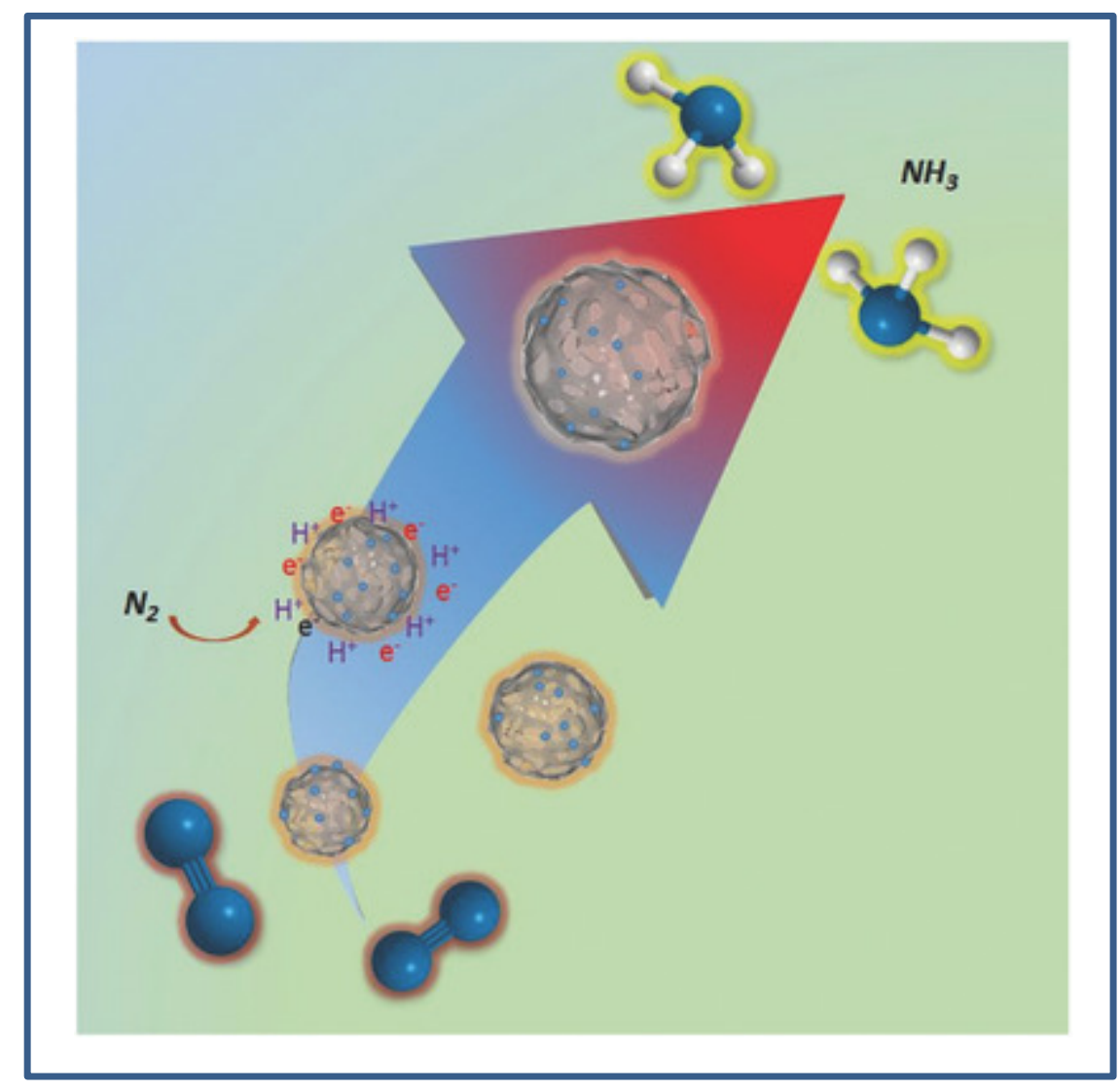


Single-site gold catalysts on hierarchical N-doped porous noble carbon for enhanced electrochemical reduction of nitrogen

Qing Qin, Tobias Heil, Markus Antonietti and Martin Oschatz*

Q. Qin, Dr. T. Heil, Prof. Dr. Dr. M. Antonietti, Dr. M. Oschatz

Max Planck Institute of Colloids and Interfaces

Department of Colloid Chemistry

Am Mühlenberg 1, 14476 Potsdam

Germany

E-mail: martin.oschatz@mpikg.mpg.de

Keywords: nitrogen fixation, single sites, N-doped carbon, gold, ammonia synthesis

Electrocatalytic reduction of nitrogen $\left(\mathrm{N}_{2}\right)$ at ambient conditions is an alternative strategy to produce ammonia $\left(\mathrm{NH}_{3}\right)$ to complement the commonly applied Haber-Bosch process. However, the achievement of high Faradaic efficiencies with high $\mathrm{NH}_{3}$ yield is still challenging. Here, we report $\mathrm{Au}$ single sites stabilized on $\mathrm{N}$-doped porous and highly oxidizing ("noble") carbons catalysts show excellent performance in $\mathrm{N}_{2}$ electroreduction. At a potential of $-0.2 \mathrm{~V}$ vs. reversible hydrogen electrode (RHE), a stable $\mathrm{NH}_{3}$ yield of $2.32 \mu \mathrm{g} \mathrm{h}^{-1}$ $\mathrm{cm}^{-2}$ is produced at an acceptable Faradaic efficiency of $12.3 \%$. Besides, there is no notable fluctuation of Faradaic efficiency and $\mathrm{NH}_{3}$ yield in 6-cycle test which indicates good stability. This work opens up new insights to improve $\mathrm{N}_{2}$ fixation performance by introducing active single sites into noble carbon catalysts for $\mathrm{N}_{2}$ electroreduction.

$\mathrm{NH}_{3}$ is the largest chemical product on earth and can be for instance used as agricultural fertilizer, chemical feedstock and hydrogen carrier. ${ }^{[1]}$ For more than 100 years the large scale production of $\mathrm{NH}_{3}$ is carried out by the Haber-Bosch process which is however a world-scale operation to be run economically. Ammonia production via a smaller, decentral process that can be carried out in less complex reactors, with simpler ammonia product separation, and 
without the need for prior synthesis gas production, is highly desirable. The key for efficiency of such alternative processes is efficient $\mathrm{N}_{2}$ fixation and so far, numerous efforts have been dedicated to this. ${ }^{[2]}$ Due to its low cost and potential use of sustainable energy, electrochemical $\mathrm{N}_{2}$ reduction reaction (NRR) at ambient conditions attracts increasing interests because it can overcome the high temperature and pressure range needed for the industrial Haber-Bosch process. ${ }^{[3]}$ Currently, some electrocatalysts have been developed in NRR, including noble metal, ${ }^{[4]}$ non-noble metal-based materials ${ }^{[5]}$ and conducting metalphthalocyanine complexes. ${ }^{[6]}$ However, the inertness of $\mathrm{N}_{2}$ with high bond dissociation energy $\left(941 \mathrm{~kJ} \mathrm{~mol}^{-1}\right)$ makes it difficult to be activated. Moreover, there is a fierce competition between NRR with hydrogen evolution reaction (HER) due to their nearly similar redox potential (0.092 V and $0.00 \mathrm{~V}$ vs. RHE for NRR and HER, respectively), which usually lead to low $\mathrm{NH}_{3}$ yields and Faradaic efficiencies. Besides a high electric conductivity, a good NRR catalyst has to provide strong $\mathrm{N}_{2}$ adsorption and activation capacity to get decent ammonia production. Based on previous research, $\mathrm{Au}$ is considered as one of the most effective catalysts for NRR due to its high affinity and ability to activate $\mathrm{N}_{2}$ for electrochemical reduction as well as its generally low tendency of hydrogen formation. ${ }^{[4 a, 4 b, 6-7]}$

One effective strategy to achieve higher Faradaic efficiency is to create more exposed active sites per unit mass of the catalysts. Single sites catalysts could meet this demand because of their theoretically perfect atom utilization for substrate adsorption and activation. However, such single sites require a suitable substrate on which they can be stabilized. N-doped porous carbons (NDPCs) are a particularly attractive substrate for the formation of single sites due to their high surface area, high porosity, and abundant $\mathrm{N}$ species which provide large possibilities to anchor and stabilize single sites. ${ }^{[8]}$ In contrast to heteroatom-free porous carbons, NDPCs stand out due to their "noble" character (i.e, they can oxidize material rather than being oxidized) and can thus stabilize single metal sites even at high loadings. ${ }^{[7,9]}$ On the other hand, such highly polarizable porous supports can improve the $\mathrm{N}_{2}$ adsorption and mass 
transfer as well. ${ }^{[10]}$ It has been recently found that $\mathrm{N}$ species in the NDPCs can facilitate the dissociation of $\mathrm{N}_{2}{ }^{[11]}$ Especially in the presence of additional single metal Lewis acidic ions, electron-rich nitrogen and/or carbon atoms within noble carbons can act as Lewis basic sites, leading to a frustrated Lewis pair (FLP) and potentially strong activation of $\mathrm{N}_{2}$ molecules by their strong electronic polarization. ${ }^{[12]}$ To the best of our knowledge, the experimental investigation of single sites fixed at NDPCs supports in electrochemical NRR has not been reported yet. Here, we report a novel catalyst composed of Au single sites supported on Ndoped carbons (denoted as AuSAs-NDPCs), which is characterized by a charming and stable NRR performance at comparably high $\mathrm{NH}_{3}$ production rate.

NDPCs were first synthesized by a template-assisted method. In a typical synthesis, hexaazatriphenylene-hexacarbonitrile (HAT) was coated on $\mathrm{SiO}_{2}$ nanospheres (Figure S1) by dispersing both in a mixture of acetonitrile and water. The light yellow solid was then collected by centrifugation after stirring at room temperature for 6 hours. Condensation of HAT under $\mathrm{N}_{2}$ flow at $900{ }^{\circ} \mathrm{C}$ and subsequent wet-chemical silica removal resulted in hollow, foam like catalytic frames with ultrathin N-doped carbon walls and good accessibility of all potential catalytic sites (Figure S2). The homogeneous distribution of carbon and nitrogen is demonstrated by the energy-dispersive X-ray spectroscopy (EDX) mapping (Figure S3). A N content of $9.2 \mathrm{wt} . \%$ is determined by elemental analysis. X-ray diffraction (XRD) patterns (Figure S4a) exhibit only two broad carbon peaks at $26^{\circ}(002)$ and $44^{\circ}(101)$, which demonstrated the high purity and amorphous phase of the as-obtained HAT-carbon which is in agreement with our previous study. ${ }^{[13]} \mathrm{N}_{2}$ physisorption experiments show a specific surface area (SSA) of around $979 \mathrm{~m}^{2} \mathrm{~g}^{-1}$ (Figure S4b). The total pore volume is $0.66 \mathrm{~cm}^{3} \mathrm{~g}^{-1}$. The shape of the isotherm with significant $\mathrm{N}_{2}$ uptakes at low and high relative pressure clearly show the hierarchical character of the NDPCs including narrow micropores from tectonically controlled HAT condensation and larger pores from the silica template spheres. All of the 
above mentioned of NDPCs provides innate advantages for the further coating of single sites and electrocatalysis.

AuSAs-NDPCs were synthesized by a simple impregnation method. ${ }^{[14]}$ As-prepared NDPCs were mixed with a $\mathrm{HAuCl}_{4}$ aqueous solution under continuous stirring for $7 \mathrm{~h}$ followed by subsequent annealing at $250{ }^{\circ} \mathrm{C}$ for 1 hour under reductive $\mathrm{Ar} / \mathrm{H}_{2}$ atmosphere. The morphology of AuSAs-NDPCs was characterized by high-angle annular dark-field scanning transmission electron microscopy (HAADF-STEM). As-obtained catalysts still show the open spherical porous structure of the initial NDPCs (Figure 1a), but no obvious Au nanoparticles or clusters can be observed (Figure 1b-c). Moreover, according to the high magnification STEM images (Figure 1d), isolated bright dots (Au single sites) are well-dispersed on the surface of NDPCs (Figure S5 for more details). The actual mass percentage of Au in AuSAsNDPCs is 0.205 wt. $\%$, which can be detected by inductively coupled plasma-atomic emission spectrometry (Table S1). In addition, the absence of crystalline Au is confirmed by the XRD patterns (Figure S4a), while the SSA of the NDPCs remains unchanged after Au decorating (Figure S4b). When the Au mass loading was increased to 0.713 wt.\% (Table S1), Au nanoparticles with the size of around 10-20 nm emerged (Figure S6a), and their presence can be further confirmed by the presence of a diffraction peak corresponding to metallic $\mathrm{Au}(111)$ after reduction (Figure S6b).

X-ray photoelectron spectroscopy (XPS) measurements of Au 4f in AuSAs-NDPCs (Figure S7) demonstrate the existence of Au species with a binding energy of electrons higher than in the metallic Au particles of AuNPs-NDPCs.${ }^{[15]}$ That means that noble NDPCs are stronger electron acceptors than Au ions. i.e., they do indeed have a higher standard electrode potential than Au. We imagine that the Au may not be fully ionized but that the electron density on these single sites is still lower than on an $\mathrm{Au}$ atom but higher than on an $\mathrm{Au}$ ion due to electron transfer from the Au "cation" to the support "anion". Deconvolution of the C 1s line scans (Figure S8a) reveals the presence of graphitic C-C carbons at $\sim 284 \mathrm{eV}, \mathrm{sp}^{2}$-hybridized 


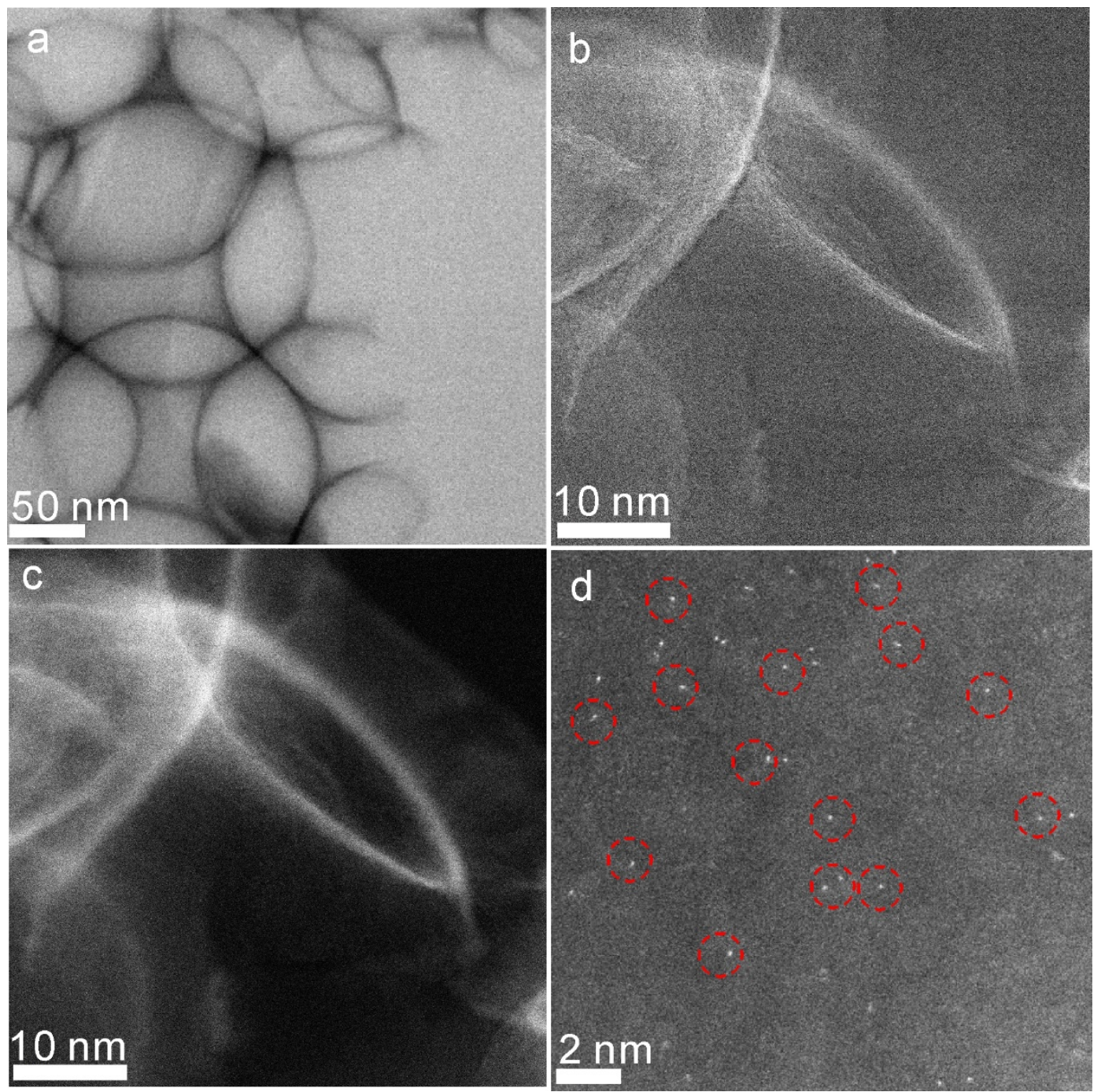

Figure 1. (a) Representative TEM image of AuSAs-NDPCs. (b) Aberration-corrected STEM image in bright field mode. (c) Aberration-corrected STEM image in dark field mode. (d) High magnification HAADF-STEM image, showing that only Au single species are present. 
carbons bounded to $\mathrm{N}$ atoms at $\sim 286 \mathrm{eV}$, and oxidized carbon atoms at $\sim 290 \mathrm{eV}$, with the binding energy all shift to slightly higher values in presence of single Au sites, as compared with NDPCs. Considering the low over-all Au content, this shift is remarkable. The same phenomenon is also observed in N 1s spectra (Figure S8b), which can be assigned to cyano$(\sim 398 \mathrm{eV})$, pyrazine groups $(\sim 399 \mathrm{eV})$, quaternary $\mathrm{N}$ atoms $(\sim 400-401 \mathrm{eV})$ and oxidized $\mathrm{N}$ atoms $(\sim 402-403 \mathrm{eV}) .{ }^{[16]}$ Thus, the lower electron density of the $\mathrm{C}$ and $\mathrm{N}$ atoms in AuSAsNDPCs indicate the strong electronic interaction of $\mathrm{Au}$ atoms with $\mathrm{C}$ and $\mathrm{N}$ atoms. ${ }^{[17]}$ To further confirm the importance of heteroatom-sites, nitrogen-free salt-templated carbons (STCs) with SSA of $1130 \mathrm{~m}^{2} \mathrm{~g}^{-1}$ (Figure S9a) were used as reference supports instead of Ndoped carbons. Unlike in NDPCs, obvious Au nanoparticles appeared (Figure S9c) at almost similar Au loading (the actual loading) as in AuSAs-NDPCs (Table S1), in line with XRD results (Figure S9b). Based on these results, the combination of large surface area and abundant acessible $\mathrm{N}$ species are crucial for the deposition and stabilization of Au single sites on the carbon supports.

In view of the unique structure of AuSAs-NDPC, we investigated its NRR performance by using a two-compartment cell with three-electrode system. AuSAs-NDPCs catalysts were first coated on carbon paper as a working electrode. Linear sweep voltammetry (LSV) tests were performed in $\mathrm{Ar}$ and $\mathrm{N}_{2}$-saturated $0.1 \mathrm{M} \mathrm{HCl}$ aqueous solution $(\mathrm{pH}=1)$. The LSV curve in $\mathrm{N}_{2}$ clearly differs from that in Ar (Figure 2a), which means that there is a significant response of the AuSAs-NDPCs electrode to $\mathrm{N}_{2}$. Furthermore, controlled electrolyses (Figure 2b) were performed under different given potentials for $2 \mathrm{~h}$ to quantify the product concentration by $\mathrm{UV}$-Vis spectroscopy after recording $\mathrm{NH}_{4}^{+}$calibration curves (Figure $\mathrm{S} 10$ ). $\mathrm{NH}_{3}$ yields and corresponding Faradaic efficiencies have been calculated (Figure 2c). An ammonia production rate of $2.32 \mu \mathrm{g} \mathrm{h}^{-1} \mathrm{~cm}^{-2}$ with the maximum Faradaic efficiency of $12.3 \%$ for $\mathrm{NH}_{3}$ was achieved at $-0.2 \mathrm{~V}$ vs. RHE, as shown in the corresponding optical image by an obvious color change before and after electrolysis (Figure S11). Regarding the very low local 

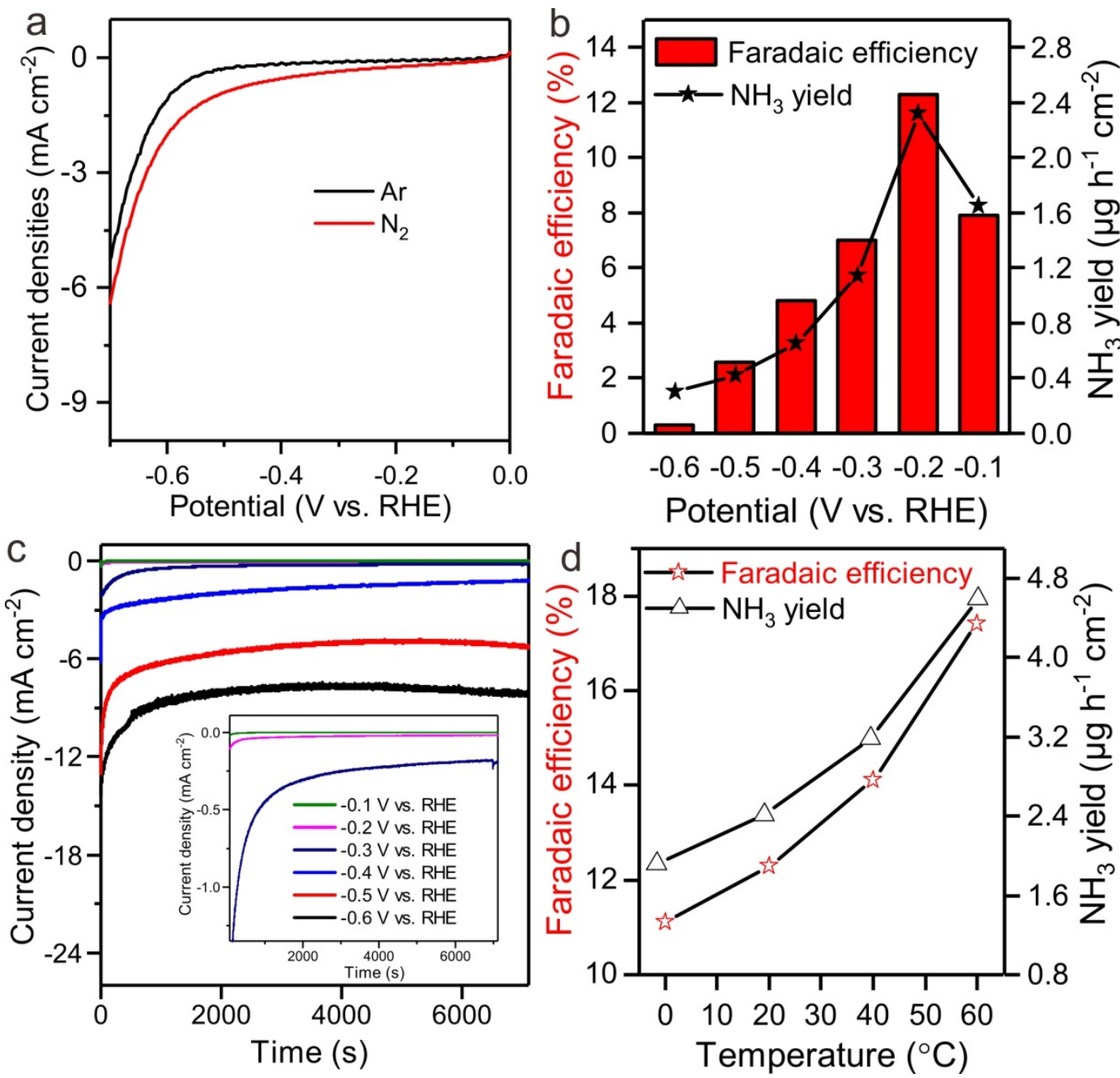

Figure 2. Electrocatalytic NRR performance of AuSAs-NDPCs electrode. a) LSV curves in an $\mathrm{N}_{2}$ saturated (black line) and Ar saturated in aqueous solution of $0.1 \mathrm{M} \mathrm{HCl}$ under ambient conditions. (b) Faradaic efficiency (red) and yield rate of $\mathrm{NH}_{3}$ at each given potential. (c) Chronoamperometry results at the corresponding potentials. (d) Faradaic efficiency and $\mathrm{NH}_{3}$ yield plotted against temperature of the electrocatalytic test at $-0.2 \mathrm{~V}$ vs. RHE under ambient pressure and different temperatures. 

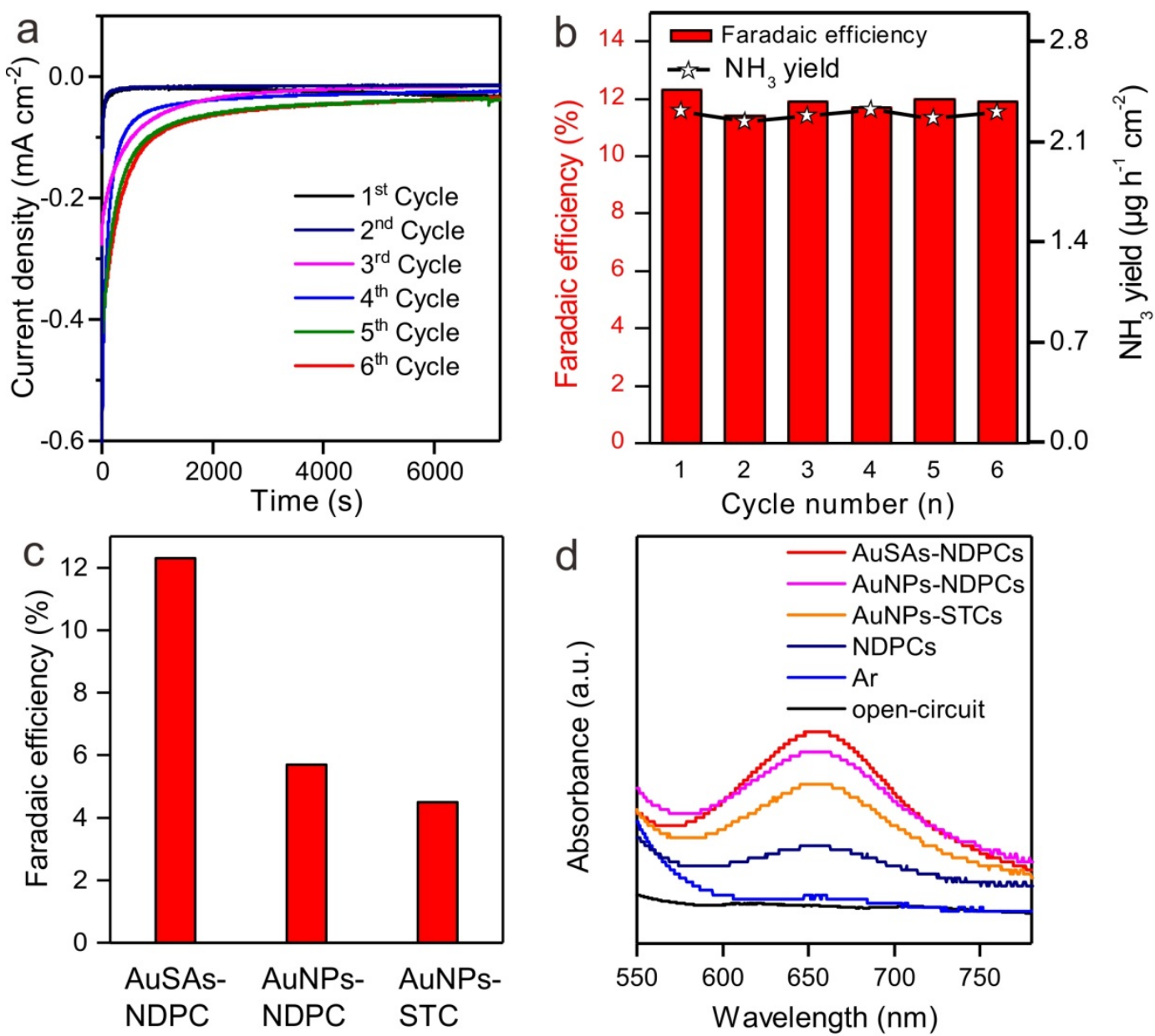

Figure 3. (a) Chronoamperometry results of AuSAs-NDPCs electrode for a stability test recorded at the potential of $-0.2 \mathrm{~V}$ vs. RHE. (b) Faradaic efficiency and $\mathrm{NH}_{3}$ yield during the recycling test. (c) Faradaic efficiency of $\mathrm{NH}_{3}$ with different catalysts at $-0.2 \mathrm{~V}$ vs. RHE. (d) UV-Vis absorption spectra of the $0.1 \mathrm{M} \mathrm{HCl}$ solution with indophenol indicator after charging at $-0.2 \mathrm{~V}$ vs. RHE for $2 \mathrm{~h}$ under various conditions. 
concentration of $\mathrm{N}_{2}$ caused by its low solubility in water which could even cause this reaction to be in a diffusion controlled mode, these Faradaic efficiencies and $\mathrm{NH}_{3}$ yield is remarkable. Besides, there is no $\mathrm{NH}_{3}$ produced from Ar-saturated electrolyte from $-0.1 \mathrm{~V}$ to $-0.6 \mathrm{~V}$ vs. RHE (Figure S12), indicating $\mathrm{NH}_{3}$ formation solely from $\mathrm{N}_{2}$ dissolved into the electrolyte and not from the $\mathrm{N}_{2}$ in the AuSAs-NDPCs electrode. With the increase of negative potential, Faradaic efficiency and $\mathrm{NH}_{3}$ yield largely decreased due to the predominance of HER, which was also demonstrated by the sharply increasing current densities in LSV curves. ${ }^{[18]}$ NRR under different temperatures was also conducted at $-0.2 \mathrm{~V}$ vs. RHE (Figure S13a). Faradaic efficiency and $\mathrm{NH}_{3}$ yield both increase with increasing reaction temperature (Figure 2d) due to higher reaction rate according to the Arrhenius equation. ${ }^{[3 c]}$ The activation energy is about 12.3 $\mathrm{KJ} \mathrm{mol}^{-1}$ (Figure S13b), which is much lower than that of traditional Haber-Bosch process $\left(57 \mathrm{~kJ} \mathrm{~mol}^{-1}\right.$ for advanced $\mathrm{Co}_{3} \mathrm{Mo}_{3} \mathrm{~N}$ catalysts). ${ }^{[19]}$ Besides the good activity, stability is also really important to value an electrocatalyst. After a recycling test for 6 times (Figure 3a), there is no obvious decrease of Faradaic efficiency and $\mathrm{NH}_{3}$ yield for AuSAs-NDPCs (Figure $3 b$ ). Chronoamperometry test at $-0.2 \mathrm{~V}$ vs. RHE for 50000 seconds and the LSV curve after it show no obvious current densities decrease (Figure S14), while TEM clearly shows that $\mathrm{Au}$ is still present as single species with no significant agglomeration after this test (Figure $\mathrm{S} 15$ ). $\mathrm{NH}_{3}$ production over reaction time also demonstrates the good stability of as obtained AuSAs-NDPCs (Figure S16).

To better understand the specific advantages of Au single sites, the NRR test was also conducted for AuNPs-NDPCs and pure NDPCs. Compared with AuSAs-NDPCs, Faradaic efficiency of AuNPs-NDPCs catalysts decreases to $5.7 \%$ at $-0.2 \mathrm{~V}$ vs. RHE (Figure 3c). Likewise, as-prepared AuNPs-STC also shows a decreased Faradaic efficiency of $4.5 \%$ at the same potential (Figure 3c). Metal-free NDPCs without $\mathrm{Au}$ could only reach the highest Faradaic efficiency of $2.1 \%$ at the potential of $-0.6 \mathrm{~V}$ vs. RHE (Figure S17). The corresponding UV-Vis adsorption spectra of catalytic products (Figure 3d) further confirm the 
excellent performance of AuSAs-NDPCs. In order to verify that $\mathrm{NH}_{3}$ was produced from NRR on AuSAs-NDPCs catalysts, as shown in Figure S18, pure carbon paper without any catalysts coating was tested in Ar and $\mathrm{N}_{2}$-saturated solution at $-0.2 \mathrm{~V}$ vs. RHE. $\mathrm{No} \mathrm{NH}_{4}^{+}$has been detected based on the UV-Vis analysis. The same result was also obtained in $\mathrm{N}_{2}$ saturated solution at open-circuit (Figure 3d).

Base on above analysis, NDPCs and Au single sites both play crucial roles in the excellent NRR performance. NDPCs, as a highly open porous structure with high surface area, can create a favourable access for active sites and mass transport during the electrochemical NRR. On the other hand, they also stabilize $\mathrm{Au}$ single sites due to the abundant $\mathrm{N}$ and $\mathrm{C}$ species with highly oxidizing character and strong polarization. It is worth to note that hydrazine $\left(\mathrm{N}_{2} \mathrm{H}_{4}\right)$ as a normal intermediate product is easily produced in electrochemical NRR. ${ }^{[20]}$ However, there is no color change before and after electrolysis under $-0.2 \mathrm{~V}$ vs. RHE by adding $\mathrm{N}_{2} \mathrm{H}_{4}$ color reagent (Figure $\mathrm{S} 19$ ). UV spectra confirm that no $\mathrm{N}_{2} \mathrm{H}_{4}$ is produced in our system under different potentials (Figure S20). Based on our above analysis, from a mechanistic point of view, $\mathrm{N}_{2}$ may firstly absorb on the surface of catalysts by interacting with (positively polarized) $\mathrm{Au}$ single species and (negatively polarized) $\mathrm{N}$ or $\mathrm{C}$ atoms. In other words, $\mathrm{N}_{2}$ is bridged into an FLP-like structure motive in the catalyst. Then activated $\mathrm{N}_{2}$ undergoes hydrogenation independently by reacting with activated $\mathrm{H}$ in the solution to form $\mathrm{N}-\mathrm{H}$ bond. ${ }^{[21]}$ As the reaction proceeds, $\mathrm{N} \equiv \mathrm{N}$ bond will be broken and $\mathrm{NH}_{3}$ is formed as the final product.

In summary, a novel catalyst construction composed of Au single atoms decorated on the surface of NDPCs was reported. The introduction of Au single atoms leads to active reaction sites, which are stabilized by N species in NDPCs. Thus, the interaction within as-prepared AuSAs-NDPCs catalysts enabled promising performance for electrochemical NRR. We believe that this work provides new opportunities for catalyst design in order to achieve efficient $\mathrm{N}_{2}$ fixation at ambient conditions by utilizing recycled electric energy. As a rather 
personal site conclusion we also believe that (in addition to enhancement of the catalysts themselves) a major step forward in the efficiency of $\mathrm{N}_{2} \mathrm{RR}$ will lie in finding ways to increase the local concentration of $\mathrm{N}_{2}$ in close proximity to the catalysts.

\section{Experimental Section}

Synthesis of $\mathrm{SiO}_{2}$ nanospheres: According to modified Stöber method reported in the literature, ${ }^{[22]}$ TEOS $(6 \mathrm{~mL})$ was rapidly injected into a mixture of ethanol $(74 \mathrm{~mL})$ and deionized water $(10 \mathrm{~mL})$, followed by the addition of ammonium aqueous solution $(28 \%, 3.15$ $\mathrm{mL}$ ). After stirring at room temperature for $1 \mathrm{~h}$, obtained colloidal suspension was washed with water and ethanol several times for further use.

Synthesis of STCs: STCs were prepared following the method described in the literature by using $\mathrm{ZnCl}_{2}$ as the salt template. ${ }^{[23]}$ In a typical procedure, sucrose $(5 \mathrm{~g}), \mathrm{ZnCl}_{2}(5 \mathrm{~g})$ and concentrated sulfuric acid $(0.55 \mathrm{~g})$ were mixed in ultrapure water $(30 \mathrm{~mL})$. The mixture was then dried at $100{ }^{\circ} \mathrm{C}$ for $6 \mathrm{~h}$ under air, followed by heating to $160^{\circ} \mathrm{C}$ and keeping for $6 \mathrm{~h}$. Asobtained product was finally transferred to a tubular furnace for carbonization at $900{ }^{\circ} \mathrm{C}$ for $2 \mathrm{~h}$ to get STCs with a heating rate of $1^{\circ} \mathrm{C} \min ^{-1}$ under $\mathrm{N}_{2}$ atmosphere.

Synthesis of NDPCs: HAT was employed as the precursor for the synthesis of STCs. In a typical process, $100 \mathrm{mg}$ of HAT and $500 \mathrm{mg}$ of $\mathrm{SiO}_{2}$ nanospheres were dispersed in a mixture of water $(15 \mathrm{~mL})$ and acetonitrile $(15 \mathrm{~mL})$ by ultrasound and stirred at room temperature for 6 h. After washing with water for several times and drying under vacuum, the light-yellow solid was carbonized at $900{ }^{\circ} \mathrm{C}$ under $\mathrm{N}_{2}$ flow with a heating rate of $4{ }^{\circ} \mathrm{C} \mathrm{min}{ }^{-1}$ for $1 \mathrm{~h}$. NDPCs were finally obtained by using aqueous hydrofluoric acid (5 wt.\%) to remove the $\mathrm{SiO}_{2}$ template.

Synthesis of AuSAs-NDPCs, AuNPs-NDPCs and AuNPs-STCs: Typically, NDPCs were dispersed in water $(15 \mathrm{~mL})$ with stirring. Then, different amount of $\mathrm{HAuCl}_{4}$ aqueous solution $(0.02 \mathrm{M})$ was slowly added into the above mixture. AuSAs-NDPCs were obtained by adding 
$0.10 \mathrm{~mL} \mathrm{HAuCl}_{4}$ aqueous solution, while $0.370 \mathrm{~mL}$ dosage resulted in the formation of AuNPs-NDPCs. After stirring at room temperature for $7 \mathrm{~h}$, the product was collected by centrifugation, washing with water and drying under vaccum at $60^{\circ} \mathrm{C}$ overnight, followed by reducing by $\mathrm{Ar} / \mathrm{H}_{2}$ atmosphere. Synthesis of AuNPs-STCs was carried out by only replacing the NDPCs with STCs.

Material characterization: The purity and crystallinity of obtained samples were characterized by X-ray powder diffraction (XRD) patterns, recorded on a Bruker D8 advance X-ray diffractometer with $\mathrm{Cu} \mathrm{Ka}$ radiation. Scanning electron microscopy (SEM) images were obtained on a LEO 1550-Gemini instrument operating at $3 \mathrm{kV}$ after sputtering with gold. Energy-dispersive X-ray (EDX) investigations were conducted on a Link ISIS-300 system equipped with a $\mathrm{Si}$ (Li) detector. Transmission electron microscopy (TEM) was conducted on JEOL ARM 200F instrument operating at $200 \mathrm{kV}$. The samples were dispersed in ethanol, and then coated on a carbon-coated copper TEM grid and finally dried at room temperature before the test. $\mathrm{N}_{2}$ physisorption isotherms measurements were carried out at $-196{ }^{\circ} \mathrm{C}$ on a Quadrasorb apparatus from Quantachrome Instruments. Prior to all measurements, the samples were outgassed at $150{ }^{\circ} \mathrm{C}$ for $20 \mathrm{~h}$ under vacuum. SSAs were calculated using the multi-point Brunauer-Emmett-Teller (BET) model in the range of $0.05<\mathrm{p} / \mathrm{p}_{0}<0.2$. X-ray photoelectron spectroscopy (XPS) measurements were performed using a Thermo Scientific $\mathrm{K}_{\text {-Alpha }}^{+}$X-ray photoelectron spectrometer. All samples were analyzed using a microfocused, monochromated Al-K $\mathrm{K}_{\alpha} \mathrm{X}$-ray source $(1486.68 \mathrm{eV} ; 400 \mu \mathrm{m}$ spot size). The K$\mathrm{Alpha}^{+}$charge compensation system was employed during analysis to prevent any localized charge buildup. The samples were mounted on conductive carbon tape and the resulting spectra analyzed using the Avantage software from Thermo Scientific.

The content of Au was analyzed by inductively coupled plasma mass spectrometry (ICP-MS). Elemental analysis was accomplished by a Vario Micro device. UV-Vis spectroscopic measurements were conducted with a Cary $50 \mathrm{UV}-\mathrm{V}$ is spectrometer. 
Electrochemical measurements: All the electrochemical measurements were performed using a Gamry potentiostat in a two-compartment cell separated by Nafion membrane, which was pretreated by heating in $5 \% \mathrm{H}_{2} \mathrm{O}_{2}$ aqueous solution at $85{ }^{\circ} \mathrm{C}$ for $1 \mathrm{~h}$ and ultrapure water for another $1 \mathrm{~h}$. Typically, working electrodes were prepared by depositing an ethanol dispersion of catalysts ink onto a carbon paper electrode $\left(1 * 1 \mathrm{~cm}^{2}\right)$ with $0.60 \mathrm{mg} \mathrm{cm}{ }^{-2}$ catalyst loading and then dried at room temperature. A platinum foil $\left(1 * 1 \mathrm{~cm}^{2}\right)$ and a saturated calomel electrode (SCE) were used as the counter and reference electrode, respectively. All the experiments were carried out in $0.1 \mathrm{M} \mathrm{HCl}$ aqueous $(\mathrm{pH}=1)$. All the potentials in this work were calculated to RHE, $\mathrm{E}(\mathrm{RHE})=\mathrm{E}(\mathrm{SCE})+0.24+0.059 * \mathrm{pH}$.

Linear sweep voltammetry (LSV) test was conducted in $\mathrm{N}_{2^{-}}$and Ar-saturated solution at a scan rate of $5 \mathrm{mV} \mathrm{s}^{-1}$. All LSV curves were steady-state ones after several cycles and directly used without additional iR correction.

Potentiostatic tests were carried out at different potentials under continuous stirring. Pure Ar or $\mathrm{N}_{2}$ was purged into the cathodic compartment for at least $30 \mathrm{~min}$ before the test, and the feed was kept during the whole test. NRR was also conducted at different temperatures $\left(0^{\circ} \mathrm{C}\right.$, $20{ }^{\circ} \mathrm{C}, 40{ }^{\circ} \mathrm{C}$ and $60{ }^{\circ} \mathrm{C}$ ) to investigate the influence of temperature on the reaction rate. Thus, activation energy can be calculated according to Arrhenius equation as follows, where A is Arrhenius constant, Ea is activation energy, $\mathrm{T}$ is reaction temperature, $\mathrm{R}$ is gas constant and $\mathrm{v}$ is corresponding reaction rate:

$$
v=A e^{-\frac{E a}{R T}}
$$

Detection of $\mathrm{NH}_{3}$ was achieved by the indophenol blue method. In detail, $2 \mathrm{~mL}$ electrolyte after potentiostatic tests was mixed with $2 \mathrm{~mL}$ of a $1 \mathrm{M} \mathrm{NaOH}$ solution containing $5 \mathrm{wt} . \%$ sodium citrate and 5 wt.\% salicylic acid. Then, $1 \mathrm{~mL}$ of $0.05 \mathrm{M} \mathrm{NaClO}$ solution was also added into the mixture, followed by addition of 1 wt. $\% \mathrm{C}_{5} \mathrm{FeN}_{6} \mathrm{Na}_{2} \mathrm{O}$ (sodium nitroferricyanide). After leaving at room temperature for $1 \mathrm{~h}$, the mixed solution was 
measured by UV-Vis spectroscopy and the adsorption peak of indophenol blue appeared at around $655 \mathrm{~nm}$. In order to accurately quantify $\mathrm{NH}_{3}$, a calibration curve was plotted by preparing a series of ammonium chloride standard solutions. Similarly, $\mathrm{N}_{2} \mathrm{H}_{4}$ was detected by Watt and Chrisp method with a color reagent containing $5.99 \mathrm{~g}$ of para-(dimenthylamino) benzaldehyde, $30 \mathrm{~mL}$ of $\mathrm{HCl}$ (concentrated) and $300 \mathrm{~mL}$ of ethanol.

Faradaic efficiency of $\mathrm{NH}_{3}$ was calculated according to the following equation:

$$
F E=\frac{3 * F * c * V}{17 * Q}
$$

where F is Faraday constant, $\mathrm{V}$ is total volume of electrolyte in cathodic compartment, $\mathrm{Q}$ is the total charge passed through the electrochemical system and $\mathrm{c}$ is the calculated concentration of $\mathrm{NH}_{3}$.

$\mathrm{NH}_{3}$ yield was also obtained according to the equation as follow:

$$
\mathrm{NH}_{3} \text { yield }=\frac{c * V}{t * A}
$$

Where $\mathrm{t}$ is reaction time, $\mathrm{A}$ is the surface area of working electrode, $\mathrm{V}$ is total electrolyte volume in cathodic compartment and c is concentration of $\mathrm{NH}_{3}$.

\section{Supporting Information}

Supporting Information is available from the Wiley Online Library or from the author.

\section{Acknowledgements}

We greatfully acknowledge financial support by the Max Planck Society. We thank Dr. Johannes Schmidt (TU Berlin, Group of Functional Materials) for providing the XPS measurements and Dr. Heike Runge for the SEM tests. Q. Q. thanks Bibi Zhou, who always shows good examples to teach her to work hard for the things that she aspires to achieve. M.O. acknowledges financial support by a Liebig Stipendium of the German Chemical Industry Fund (Fonds der Chemischen Industrie, FCI). 
References

[1] a) R. D. Milton, S. Abdellaoui, N. Khadka, D. R. Dean, D. Leech, L. C. Seefeldt, S. D. Minteer, Energy Environ. Sci. 2016, 9, 2550; b) T. Spatzal, K. A. Perez, O. Einsle, J. B. Howard, D. C. Rees, Science 2014, 345, 1620; c) D. V. Yandulov, R. R. Schrock, Science 2003, 301, 76; d) I. Čorić, B. Q. Mercado, E. Bill, D. J. Vinyard, P. L. Holland, Nature 2015, 526, 96; e) J. M. McEnaney, A. R. Singh, J. A. Schwalbe, J. Kibsgaard, J. C. Lin, M. Cargnello, T. F. Jaramillo, J. K. Nørskov, Energy Environ. Sci. 2017, 10, 1621; f) S. Licht, B. Cui, B. Wang, F. F. Li, J. Lau, S. Liu, Science 2014, 345, 637.

a) C. Guo, J. Ran, A. Vasileff, S.-Z. Qiao, Energy Environ. Sci. 2018, 11, 45; b) X. Wang, G. Yang, J. Zhang, S. Chen, Y. Wu, Q. Zhang, J. Wang, Y. Han, Y. Tan, Chem. Commun. 2016, 52, 7352.

[3] a) R. Lan, J. T. S. Irvine, S. W. Tao, Sci. Rep. 2013, 3, 1145; b) H. K. Lee, C. S. L. Koh, Y. H. Lee, C. Liu, I. Y. Phang, X. Han, C. K. Tsung, X. Y. Ling, Sci. Adv. 2018, 4, eaar3208; c) V. Kordali, G. Kyriacou, C. Lambrou, Chem. Commun. 2000, 17, 1673; d) D. Yang, T. Chen, Z. Wang, J. Mater. Chem. A 2017, 5, 18967; e) E. Y. Jeong, C. Y. Yoo, C. H. Jung, J. H. Park, Y. C. Park, J. N. Kim, S. G. Oh, Y. Woo, H. C. Yoon, ACS Sustain. Chem. Eng. 2017, 5, 9662.

[4] a) D. Bao, Q. Zhang, F. L. Meng, H. X. Zhong, M. M. Shi, Y. Zhang, J. M. Yan, Q. Jiang, X. B. Zhang, Adv. Mater. 2017, 29, 1604799; b) M. M. Shi, D. Bao, B. R. Wulan, Y. H. Li, Y. F. Zhang, J. M. Yan, Q. Jiang, Adv. Mater. 2017, 29, 1606550; c) M.-M. Shi, D. Bao, S.-J. Li, B.-R. Wulan, J.-M. Yan, Q. Jiang, Adv. Energy Mater., DOI: 10.1002/aenm.201800124.

[5] a) S. M. Chen, S. Perathoner, C. Ampelli, C. Mebrahtu, D. S. Su, G. Centi, Angew. Chem. Int. Ed. 2017, 56, 2699; b) K. Kim, N. Lee, C.-Y. Yoo, J.-N. Kim, H. C. Yoon, J.-I. Han, J. Electrochem. Soc. 2016, 163, F610; c) C. Lv, C. Yan, G. Chen, Y. Ding, J. Sun, Y. Zhou, G. Yu, Angew. Chem. Int. Ed. 2018, 130, 6181.

[6] a) F. Koleli, T. Ropke, Appl. Catal. B-Environ. 2006, 62, 306; b) G. F. Chen, X. Cao, S. Wu, X. Zeng, L. X. Ding, M. Zhu, H. Wang, J. Am. Chem. Soc. 2017, 139, 9771.

[7] O. G. A., W. Hong, W. Lu, W. Qiang, Y. Shuyang, S. Wei, S. Yue, J. Zhiping, Q. Qiao, Z. Yimei, S. Pengfei, L. Debao, H. Le, Z. Xiaohong, Y. Jiayin, W. Tom, Angew. Chem. Int. Ed., DOI: 10.1002/anie.201805514.

[8] a) B. Qiao, A. Wang, X. Yang, L. F. Allard, Z. Jiang, Y. Cui, J. Liu, J. Li, T. Zhang, Nat. Chem. 2011, 3, 634; b) G. Geng, P. Chen, B. Guan, L. Jiang, Z. Xu, D. Di, Z. Tu, W. 
Hao, Y. Yi, C. Chen, M. Liu, W. Hu, ACS Nano 2017, 11, 4866; c) J. Wan, W. Chen, C. Jia, L. Zheng, J. Dong, X. Zheng, Y. Wang, W. Yan, C. Chen, Q. Peng, D. Wang, Y. Li, Adv. Mater. 2018, 30, 1705369; d) Z. Chen, S. Pronkin, T.-P. Fellinger, K. Kailasam, G. Vilé, D. Albani, F. Krumeich, R. Leary, J. Barnard, J. M. Thomas, J. Pérez-Ramírez, M. Antonietti, D. Dontsova, ACS Nano 2016, 10, 3166.

[9] A. Markus, O. Martin, Adv. Mater. 2018, 30, 1706836.

[10] L. Qie, W. M. Chen, Z. H. Wang, Q. G. Shao, X. Li, L. X. Yuan, X. L. Hu, W. X. Zhang, Y. H. Huang, Adv. Mater. 2012, 24, 2047.

[11] a) Y.-H. Tian, S. Hu, X. Sheng, Y. Duan, J. Jakowski, B. G. Sumpter, J. Huang, The J. Phy. Chem. Lett. 2018, 9, 570; b) Y. Cao, Y. Gao, H. Zhou, X. Chen, H. Hu, S. Deng, X. Zhong, G. Zhuang, J. Wang, Adv. Theor. Simul. 2018, 1, 1800018; c) F. Pan, H. Zhang, K. Liu, D. Cullen, K. More, M. Wang, Z. Feng, G. Wang, G. Wu, Y. Li, ACS Catal. 2018, 8, 3116.

[12] D. W. Stephan, Acc. Chem. Res. 2015, 48, 306.

[13] R. Walczak, B. Kurpil, A. Savateev, T. Heil, J. Schmidt, Q. Qin, M. Antonietti, M. Oschatz, Angew. Chem. Int. Ed., DOI: 10.1002/ange.201804359.

[14] G. Vile, D. Albani, M. Nachtegaal, Z. Chen, D. Dontsova, M. Antonietti, N. Lopez, J. Perez-Ramirez, Angew. Chem. Int. Ed. 2015, 54, 11265.

[15] W. Zhang, Y. Hu, L. Ma, G. Zhu, Y. Wang, X. Xue, R. Chen, S. Yang, Z. Jin, Adv. Sci. 2018, 5, 1700275.

[16] D. J. Martin, K. Qiu, S. A. Shevlin, A. D. Handoko, X. Chen, Z. Guo, J. Tang, Angew. Chem. Int. Ed. 2014, 53, 9240.

[17] K. Kamiya, R. Kamai, K. Hashimoto, S. Nakanishi, Nat. Commun. 2014, 5, 5040.

[18] T. Oshikiri, K. Ueno, H. Misawa, Angew. Chem. Int. Ed. 2016, 128, 4010.

[19] R. Kojima, K. Aika, Appl. Catal. A-Gen 2001, 218, 121.

[20] D.-Y. Hwang, A. M. Mebel, J. Phy. Chem. A 2003, 107, 2865.

[21] J. Deng, J. A. Iñiguez, C. Liu, Joule 2018, 2, 846.

[22] X. Fang, C. Chen, Z. Liu, P. Liu, N. Zheng, Nanoscale 2011, 3, 1632.

[23] R. Yan, M. Antonietti, M. Oschatz, Adv. Energy Mater., DOI: 10.1002/aenm.201800026. 